\title{
Universiteit
}

Leiden

The Netherlands

\section{First-order nature of a metamagnetic transition and mechanism of giant magnetoresistance in Mn2Sb0.95Sn0.05}

Zhang, Y.Q.; Zhang, Z.D.; Aarts, J.

\section{Citation}

Zhang, Y. Q., Zhang, Z. D., \& Aarts, J. (2004). First-order nature of a metamagnetic transition and mechanism of giant magnetoresistance in Mn2Sb0.95Sn0.05. Physical Review B, 2005(71), 229902. doi:10.1103/PhysRevB.70.132407

Version: $\quad$ Not Applicable (or Unknown)

License: $\quad$ Leiden University Non-exclusive license

Downloaded from: https://hdl.handle.net/1887/45420

Note: To cite this publication please use the final published version (if applicable). 


\title{
First-order nature of a metamagnetic transition and mechanism of giant magnetoresistance in $\mathrm{Mn}_{2} \mathrm{Sb}_{0.95} \mathrm{Sn}_{0.05}$
}

\author{
Y. Q. Zhang and Z. D. Zhang \\ Shenyang National Laboratory for Materials Science, Institute of Metal Research, Chinese Academy of Sciences, 72 Wenhua Road, \\ Shenyang 110016, People's Republic of China \\ and International Centre for Materials Physics, Chinese Academy of Sciences, 72 Wenhua Road, Shenyang 110016, \\ People's Republic of China \\ J. Aarts \\ Kamerlingh Onnes Laboratory, Leiden University, PO Box 9504, 2300 RA Leiden, The Netherlands
}

(Received 25 February 2004; published 25 October 2004)

\begin{abstract}
Magnetization behavior across a metamagnetic transition from an antiferromagnetic state to a ferrimagnetic state is investigated in detail for compound $\mathrm{Mn}_{2} \mathrm{Sb}_{0.95} \mathrm{Sn}_{0.05}$. The study clearly brings out various generic features associated with a first-order transition, viz., the appearance of hysteresis and the coexistence of magnetic phases. We also observe that the magnetization versus field butterfly loops occurs, while the virgin curve lies outside the envelope magnetization curve. The electronic specific-heat coefficient at low temperatures increases with increasing applied magnetic field, after the field is larger than the critical transition field. This is direct evidence of the formation of a super-zone gap that yields the change of density of electric states and further proves that the large magnetoresistance effect in intermetallic compounds is originated from the reconstruction of Fermi surface due to the collapse of the super-zone gap after the metamagnetic transition.
\end{abstract}

DOI: 10.1103/PhysRevB.70.132407

The intermetallic compound $\mathrm{Mn}_{2} \mathrm{Sb}$ crystallizes in the $\mathrm{Cu}_{2} \mathrm{Sb}$-type tetragonal structure with two structurally nonequivalent cation positions I and II, which are tetrahedrally and octahedrally surrounded by anions, respectively. Mn1 and $\mathrm{Mn} 2$ atoms in these two positions have magnetic moments of different values and opposite directions. As a consequence, the $\mathrm{Mn}_{2} \mathrm{Sb}$ compound is a ferrimagnet below its Curie temperature $T_{C}=550 \mathrm{~K} .{ }^{1}$ In addition to the phase transition from a paramagnetic state to a ferrimagnetic (FI) state, compounds with substituted elements $(\mathrm{Co}, \mathrm{Cr}, \mathrm{Cu}$, and $\mathrm{V})$ for $\mathrm{Mn}$, as well as of $\mathrm{Ge}$ and $\mathrm{Sn}$ for $\mathrm{Sb}$, exhibit another phase transition to an antiferromagnetic (AF) state as the temperature decreases. ${ }^{2}$ Below the FI-AF transition temperature, a metamagnetic transition from AF to FI state can be induced by an applied external field, with which a large magnetoresistance (MR) effect is associated. ${ }^{3-5}$ In our previous work, ${ }^{3}$ the magnetotransport behavior across the metamagnetic transition from a low-field and low-temperature AF state to a high-field and high-temperature FI state was studied for $\mathrm{Mn}_{2} \mathrm{Sb}_{1-x} \mathrm{Sn}_{x}(0<x \leqslant 0.4)$ compounds. The origin of the large MR effect was discussed in terms of the reconstruction of Fermi surface, due to the collapse of a super-zone gap after the metamagnetic transition. However, no direct evidence of the formation of such super-zone gap that should yield the change of density of the electron states (DOS) in this kind of compounds has been reported so far. Moreover, no detailed demonstration has been given of the first-order nature of the metamagnetic transition. The present paper focuses on one sample with composition $\mathrm{Mn}_{2} \mathrm{Sb}_{0.95} \mathrm{Sn}_{0.05}$, among the $\mathrm{Mn}_{2} \mathrm{Sb}_{1-x} \mathrm{Sn}_{x}(0<x \leqslant 0.4)$ compounds investigated before. ${ }^{3}$ The present work deals with the first-order nature of the AF-FI transition and gives direct evidence of the formation of the super-zone gap in $\mathrm{Mn}_{2} \mathrm{Sb}_{0.95} \mathrm{Sn}_{0.05}$ compound. The study of magnetic and magnetotransport proper-
PACS number(s): 75.47.De, 75.47.Np, 75.50.Ee, 75.50.Gg

ties was limited, in our previous work, ${ }^{3}$ to the temperatures above $50 \mathrm{~K}$ and magnetic fields below $5 \mathrm{~T}$. In this work, the measurements for magnetic and magnetotransport properties on the $\mathrm{Mn}_{2} \mathrm{Sb}_{0.95} \mathrm{Sn}_{0.05}$ compound are extended to lower temperatures (down to $5 \mathrm{~K}$ ) with higher magnetic fields (up to $8 \mathrm{~T}$ ). Furthermore, the measurement of specific heat in zero field and in magnetic fields $(B=3.5$ and $4 \mathrm{~T})$ was carried out at a constant pressure $\left[C_{P}=(d Q / d T)_{P}\right]$ from 25 to $1.8 \mathrm{~K}$. This detailed study allows us to draw additional conclusions concerning the magnetic and magnetotransport properties of this kind of material. The observation of a hysteresis in magnetization and of the coexistence of different magnetic phases (through the technique of minor hysteresis loops) clearly reveals the first-order nature of the metamagnetic transition. The electric specific-heat coefficient at low temperatures increases with increasing the applied field, above the critical transition field, which gives the first direct evidence of the formation of the super-zone gap. One of the main contributions of the present work is to prove experimentally the point of view that the large MR effect in intermetallic compounds is originated from the reconstruction of Fermi surface.

The sample used in the present study belongs to the same batch of samples as used earlier in the study of magnetic and transport properties and the detailed preparation and characterization of the sample can be found elsewhere. ${ }^{3}$ We used a commercial superconducting quantum interference device magnetometer for measuring the magnetization and used a commercial measurement platform [a physical property measurement system (PPMS) from Quantum Design] for measuring the MR both as a function of temperature and magnetic field. The measurement of specific heat in zero field and in different magnetic fields was performed at a constant pressure $\left[C_{P}=(d Q / d T)_{P}\right]$ from 25 to $1.8 \mathrm{~K}$ using the PPMS. 


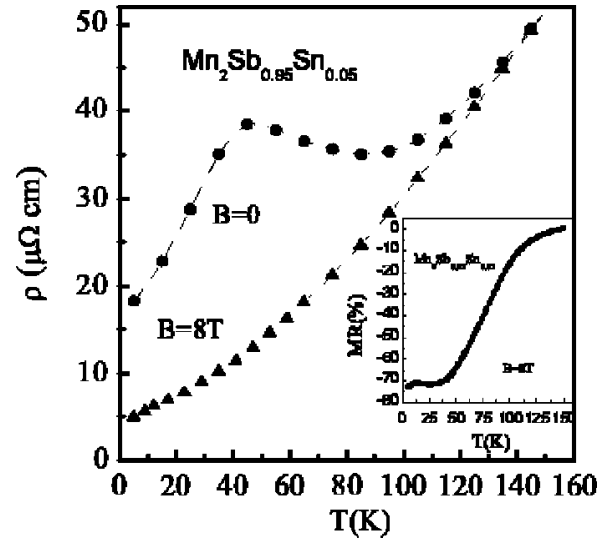

FIG. 1. Temperature dependence of the resistivity of $\mathrm{Mn}_{2} \mathrm{Sb}_{0.95} \mathrm{Sn}_{0.05}$ compound at zero field and at a magnetic field of $8 \mathrm{~T}$. The inset is for temperature dependence of the MR of $\mathrm{Mn}_{2} \mathrm{Sb}_{0.95} \mathrm{Sn}_{0.05}$ compound.

Figure 1 shows the temperature dependence of the resistivity $\rho(T)$ of $\mathrm{Mn}_{2} \mathrm{Sb}_{0.95} \mathrm{Sn}_{0.05}$ measured in zero field and in an applied field of $8 \mathrm{~T}$. In the zero field $\rho(T)$ first decreases, then rises abruptly at a certain temperature, reaches a maximum, and finally decreases with decreasing temperature. The FI-AF transition temperature $T_{\mathrm{FI}-\mathrm{AF}}$, defined as the temperature at which resistivity abruptly rises, is $85 \mathrm{~K}$ (slightly lower than the previous value of $94 \mathrm{~K}$ ). ${ }^{3}$ As reported before, ${ }^{3}$ for $\mathrm{Mn}_{2} \mathrm{Sb}_{1-x} \mathrm{Sn}_{x}(0.15<x \leqslant 0.4)$ compounds, the magnetic field shifts the $T_{\mathrm{FI}-\mathrm{AF}}$ towards lower temperatures. For the present compound $\mathrm{Mn}_{2} \mathrm{Sb}_{0.95} \mathrm{Sn}_{0.05}$, the resistance in a field of $8 \mathrm{~T}$ decreases monotonously with decreasing temperature and no $T_{\mathrm{FI}-\mathrm{AF}}$ is observed. This indicates that below the $T_{\mathrm{FI}-\mathrm{AF}}$ for the zero field, the field-induced AF-FI metamagnetic transition happens at fields less than $8 \mathrm{~T}$. A large negative magnetoresistance $\{\mathrm{MR}=[\rho(H)-\rho(0)] / \rho(0)\}$ is associated with this metamagnetic transition (see the inset of Fig. 1) and no peak is observed for the temperature dependence of the MR for $\mathrm{Mn}_{2} \mathrm{Sb}_{0.95} \mathrm{Sn}_{0.05}$.

Figure 2 compares magnetic-field dependences of MR and magnetization at $5 \mathrm{~K}$. The absolute MR starts to increase, when the metamagnetic transition occurs at the field of $1.5 \mathrm{~T}$. After the metamagnetic transition, the MR does not saturate up to the maximum applied field of $8 \mathrm{~T}$. This indi-

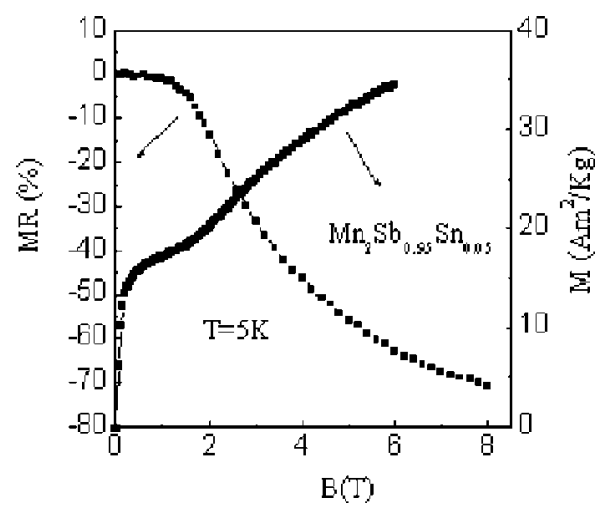

FIG. 2. Field dependence of $\mathrm{MR}$ and magnetization of $\mathrm{Mn}_{2} \mathrm{Sb}_{0.95} \mathrm{Sn}_{0.05}$ compound at $5 \mathrm{~K}$.

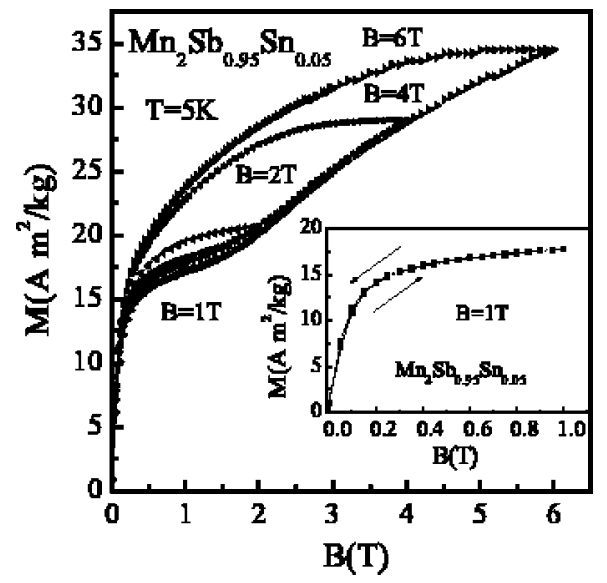

FIG. 3. MHLs initiated during both the ascending-field cycle and the descending-field cycle at the fields of 1,2, 4, and $6 \mathrm{~T}$ at $5 \mathrm{~K}$.

cates that the sample does not reach a completely ferrimagnetic state in the range of available fields. A MR as large as $-71 \%$ in the field of $8 \mathrm{~T}$ is obtained at $5 \mathrm{~K}$.

Figure 3 shows magnetization curves taken at $5 \mathrm{~K}$. The magnetization shows a sharp rise until $B=1.5 \mathrm{~T}$ indicating a field-induced AF-FI transition. The onset field $B_{M}$ of AF-FI transition is estimated as the field at which the $M-B$ curve changes the curvature from convex to concave. Below this field, the compound is in the AF state, the appearance of nonlinearity at low fields is probably due to parasitic ferromagnetism. When the field is reduced after crossing the critical value $B_{M}$, a large hysteresis is observed. A sharp rise in magnetization accompanied by a hysteresis is traditionally taken as a signature of a first-order magnetic transition. This hysteresis is different from that normally found in hard ferromagnets. The hysteresis in the case of a ferromagnet, which is due to domain wall pinning and/or anisotropy, has a maximum width at $B=0$, while in the present case the width almost goes to zero at $B=0$. Apart from the hysteresis, the other signature of the first-order transition is the coexistence of different magnetic phases. We now show the experimental observation of the phase coexistence through the technique of minor hysteresis loops (MHLs). A MHL results when the direction of a field is reversed before the transition is completed. One can initiate a minor loop on the ascending-field circle (i.e., reduce the field before the transition is completed). The minor loop initiated at a low field of $1 \mathrm{~T}$ in the field-increasing cycle on the virgin curve is completely reversible, indicating that the transition has not yet begun (see the inset in Fig. 3). As we initiate minor loops from high fields, we start to observe hysteresis, and the amount of hysteresis, increases as the value of the field for initiation of the MHL is increased. This indicates that the field-increasing curve (above $B_{M}$ ) corresponds to the transformation of the AF phase to a FI phase, with the AF phase persisting as a metastable phase over some field regime. Similarly, the fielddecreasing curve corresponds to the transformation of the FI phase to the AF phase, with the FI phase persisting as a metastable phase over some field regime. The MHLs thus show the presence of the phase coexistence in the region 


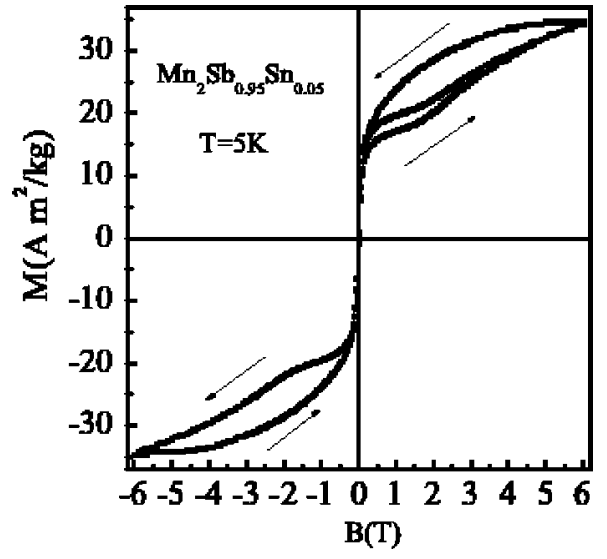

FIG. 4. Magnetization versus field butterfly loop for $\mathrm{Mn}_{2} \mathrm{Sb}_{0.95} \mathrm{Sn}_{0.05}$ compound at $5 \mathrm{~K}$.

bounded by the envelope curve. Had there been no phase coexistence, we would not have been able to produce any MHL and all the minor loops would have been reversible. This is in good agreement with the previous report, ${ }^{3}$ in which we can see the phase coexistence clearly from the temperature dependence of MR in a fixed applied field, the field dependence of MR and magnetization in different temperatures.

The hysteresis loop collapses before $B=0$ and reappears in the third quadrant when the field crosses $B=0$ in the negative direction, resulting in a butterfly loop (see Fig. 4). Such double loops have been observed in ferroelectrics, and it has been conclusively shown that only a first-order transition can explain this feature. ${ }^{6}$ We are unaware of similar arguments in the case of AF-FI transition. Moreover, clearly shown in Fig. 4, a virgin curve lies outside the envelope curve during a first-order AF-FI transition. Such a relation between the virgin curve and the envelope curve is quite uncommon in magnetic materials except for some granular materials ${ }^{7,8}$ in which the surface-to-volume ratio plays an important role and in Fe-Co-Ni alloys. ${ }^{9}$ In the latter case, no explanation has been given for such an anomalous relation between the virgin and envelope curve. In our bulk material, the surface-to-volume ratio is definitely much smaller than was the case in Refs. 7 and 8 ; in addition, the magnetic properties of our material is different from those of $\mathrm{Fe}-\mathrm{Co}-\mathrm{Ni}$ alloys. A similar phenomenon was observed in $\mathrm{Ce}\left(\mathrm{Fe}_{0.96} \mathrm{Al}_{0.04}\right)_{2}$, where an AF-F (ferromagnetic) transition happens. ${ }^{10}$

Figure 5 shows results of the measurement of specific heat $C_{P}$. the linear curves allow a determination value of the electronic specific heat coefficient $\gamma$ from the relation $C_{P}$ $=\gamma T+b T^{3}$. It can be seen that the substitution of $\mathrm{Sn}$ for $\mathrm{Sb}$ leads to the significant decrease of $\gamma$ from $40 \mathrm{~mJ} \mathrm{~K}^{-2} \mathrm{~mol}^{-1}$ for $\mathrm{Mn}_{2} \mathrm{Sb}$ to $32 \mathrm{~mJ} \mathrm{~K}^{-2} \mathrm{~mol}^{-1}$ for $\mathrm{Mn}_{2} \mathrm{Sb}_{0.95} \mathrm{Sn}_{0.05}$. This change in the $\gamma$ can be connected with a change in the type of magnetic ordering. ${ }^{11-13}$ The substitution of $\mathrm{Sb}$ by $\mathrm{Sn}$ atoms in the nonmagnetic sublattice leads to the quantitative redistribution of the sublattice magnetization Mn1 and Mn2 and the transition from FI to AF state. ${ }^{2,3}$ Moreover, with increasing Sn concentration, the AF exchange interaction of

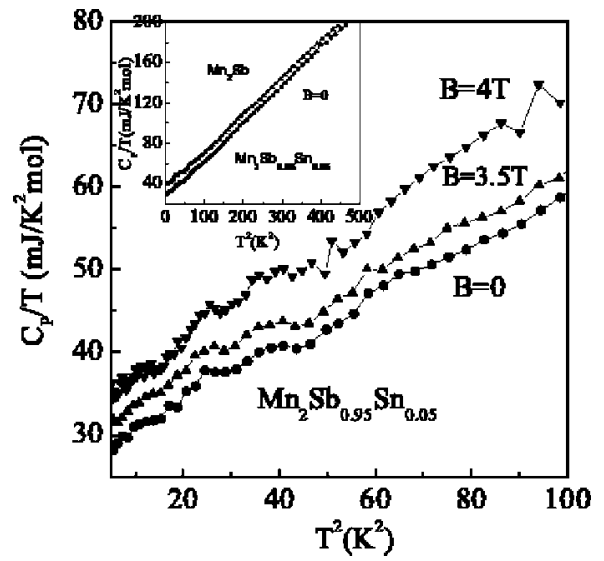

FIG. 5. $C_{P} / T$ versus $T^{2}$ for $\mathrm{Mn}_{2} \mathrm{Sb}_{0.95} \mathrm{Sn}_{0.05}$ compound in different fields. The inset represents $C_{P} / T$ versus $T^{2}$ for $\mathrm{Mn}_{2} \mathrm{Sb}$ and $\mathrm{Mn}_{2} \mathrm{Sb}_{0.95} \mathrm{Sn}_{0.05}$ compounds in zero fields.

Mn1 and Mn2 becomes stronger and shifts the phase transition temperature to higher temperatures. However, no direct evidence of the formation of the super-zone gap in this compound, which can connect the change of electric state density with magnetic transition, has been reported so far. Figure 5 shows the $T^{2}$ dependence of $C_{P} / T$ for compound $\mathrm{Mn}_{2} \mathrm{Sb}_{0.95} \mathrm{Sn}_{0.05}$ in different fields. At 3.5 T, $\gamma$ has evidently increased, and at $4 \mathrm{~T}, \gamma$ continues the increase. This indicates that as the applied field passes the critical field of the AF-FI transition, the electronic specific-heat coefficient $\gamma$ increases with increasing the field. This means that when the metamagnetic transition occurs, the electronic specific-heat coefficient $\gamma$ starts to increase. These results illustrate clearly that the super-zone gap is formed in the AF state, leading to the decrease in the DOS near the Fermi level, and when ferromagnetic state is recovered by the metamagnetic transition, the DOS at Fermi level increases. The results prove further that the large MR effect in this type of compound is originated from the reconstruction of Fermi surface, due to the collapse of the super-zone gap after the metamagnetic transition.

In conclusion, we have investigated magnetization behavior in detail across a metamagnetic transition from AF to FI state in the $\mathrm{Mn}_{2} \mathrm{Sb}_{0.95} \mathrm{Sn}_{0.05}$ compound. The study clearly brings out various generic features associated with a firstorder transition; viz., the appearance of hysteresis, the coexistence of magnetic phases. We observe as well that the magnetization versus field butterfly loops occurs, while the virgin curve lies outside the envelope magnetization curve. Lowtemperature specific-heat measurement indicates that the electric specific-heat coefficient increases with increasing the applied field, when the field is larger than the critical transition field. This is the first direct evidence of the formation of the super-zone gap, which further proves that the large MR effect in intermetallic compounds is originated from the reconstruction of Fermi surface, due to the collapse of the super-zone gap after the metamagnetic transition.

This work has been supported by the National Natural Science Foundation of China under Grant No. 50332020. 
${ }^{1}$ J. H. Wijngaard, C. Haas, and R. A. de Groot, Phys. Rev. B 45, 5395 (1992).

${ }^{2}$ V. M. Ryzhkovsk, V. P. Dymont, and Z.L. Erofeenko, Phys. Status Solidi A 130, 163 (1992).

${ }^{3}$ Y.Q. Zhang and Z. D. Zhang, Phys. Rev. B 67, 132405 (2003).

${ }^{4}$ Y.Q. Zhang, Z. D. Zhang, D.K. Xiong, W.F. Li, and J. He, J. Appl. Phys. 94, 4726 (2003).

${ }^{5}$ Y. Nagata, T. Hagii, S. Yashiro, H. Samata, and S. Abe, J. Alloys Compd. 292, 11 (1999).

${ }^{6}$ A. J. Dekkar, Solid State Physics (Macmillan, New Delhi, 1985).

${ }^{7}$ R. D. Zysler, C. A. Ramos, E. De Biasi, H. Romero, A. Ortega, and D. Fiorani, J. Magn. Magn. Mater. 221, 37 (2000).

${ }^{8}$ E. Tronc, A. Ezzir, R. Cherkaoui, C. Chanéac, M. Noguès, H.
Kachkachi, D. Fiorani, A. M. Testa, J. M. Grenèche, and J. P. Jolivet., J. Magn. Magn. Mater. 221, 63 (2000).

${ }^{9}$ R. M. Bozorth, Ferromagnetism (IEEE, Piscataway, NJ, 1994).

${ }^{10}$ M. Manekar, S. Chaudhary, M. K. Kanwal, J. Singh, S. B. Roy and P. Chaddah, J. Phys.: Condens. Matter 14, 4477 (2002).

${ }^{11}$ N.Y. Baranov, Yu. A. Khrulev, M. I. Bartashevich, T. Goto, H. Aruga Katori, G. Hilscher, and W. Perthold, J. Magn. Magn. Mater. 140-144, 1543 (1995).

${ }^{12}$ M.I. Bartashevich, T. Goto, T. Tomita, N.V. Baranov, S.V. Zemlyanski, G. Hilscher, and H. Michor, Physica B 318, 198 (2002).

${ }^{13}$ N.V. Baranov, P.E. Markin, E. Gratz, G. Hilscher, and R. Resel, J. Alloys Compd. 187, L7 (1992). 